This is a post-peer-reviewed, pre-copyedit version of an article published in Lecture Notes in Computer Science. The final authenticated version is available online at: http://dx.doi.org/10.1007/978-3-319-44543-4_18

\title{
A Boundary Property for Upper Domination
}

\author{
Hassan AbouEisha ${ }^{1}$, Shahid Hussain ${ }^{1}$, Vadim $\operatorname{Lozin}^{2(\bowtie)}{ }^{(}$, Jérôme Monnot ${ }^{3}$, \\ Bernard Ries ${ }^{4}$, and Viktor Zamaraev ${ }^{2}$ \\ 1 King Abdullah University of Science and Technology, Thuwal, Saudi Arabia \\ \{hassan.aboueisha, shahid.hussain\}@kaust.edu.sa \\ 2 Mathematics Institute, University of Warwick, Coventry CV4 7AL, UK \\ $\{$ V.Lozin, V.Zamaraev\}@warwick.ac.uk \\ 3 \\ CNRS, LAMSADE UMR 7243, University Paris-Dauphine, Paris, France \\ jerome.monnot@dauphine.fr \\ 4 Department of Informatics, University of Fribourg, Bd de Pérolles 90, \\ 1700 Fribourg, Switzerland \\ bernard.ries@unifr.ch
}

\begin{abstract}
An upper dominating set in a graph is a minimal (with respect to set inclusion) dominating set of maximum cardinality. The problem of finding an upper dominating set is generally NP-hard, but can be solved in polynomial time in some restricted graph classes, such as $P_{4}$-free graphs or $2 K_{2}$-free graphs. For classes defined by finitely many forbidden induced subgraphs, the boundary separating difficult instances of the problem from polynomially solvable ones consists of the so called boundary classes. However, none of such classes has been identified so far for the upper dominating set problem. In the present paper, we discover the first boundary class for this problem.
\end{abstract}

\section{Introduction}

In a graph $G=(V, E)$, a dominating set is a subset of vertices $D \subseteq V$ such that any vertex outside of $D$ has a neighbour in $D$. A dominating set $D$ is minimal if no proper subset of $D$ is dominating. An upper dominating set is a minimal dominating set of maximum cardinality. The UPPER DOMINATING SET problem (i.e. the problem of finding an upper dominating set in a graph) is known to be NP-hard [5]. Moreover, it remains difficult under substantial restrictions, for instance, for triangle-free graphs and the complements of bipartite graphs [1]. On the other hand, in some particular graph classes, the problem can be solved in polynomial time, which is the case for bipartite graphs [6], chordal graphs [10], generalized series-parallel graphs [9], graphs of bounded clique-width [7] and $2 K_{2}$-free graphs [1]. What other restrictions are necessary and sufficient for polynomial-time solvability of the problem? For classes defined by finitely many forbidden induced subgraphs, this question can be answered through the notion of boundary classes. This notion was introduced in [2] to study the MAXIMUM

V. Lozin - The author gratefully acknowledges support from EPSRC, grant $\mathrm{EP} / \mathrm{L} 020408 / 1$. 
INDEPENDENT SET problem and was later applied to many other algorithmic graph problems (see e.g. [3,4,13]). However, for the UPPER DOMINATING SET problem no boundary classes have been identified so far. In the present paper, we reveal the first boundary class for this problem.

The organization of the paper is as follows. In Sect.2, we introduce basic definitions, including the notion of a boundary class, and prove some preliminary results. Section 3 contains the main result of the paper. Finally, in Sect. 4 we discuss an open problem.

\section{Preliminaries}

We denote by $\mathcal{G}$ the set of all simple graphs, i.e. undirected graphs without loops and multiple edges. The girth of a graph $G \in \mathcal{G}$ is the length of a shortest cycle in $G$. As usual, we denote by $K_{n}, P_{n}$ and $C_{n}$ the complete graph, the chordless path and the chordless cycle with $n$ vertices, respectively. Also, $\bar{G}$ denotes the complement of $G$. A star is a connected graph in which all edges are incident to a same vertex, called the center of the star.

Let $G=(V, E)$ be a graph with vertex set $V$ and edge set $E$, and let $u$ and $v$ be two vertices of $G$. If $u$ is adjacent to $v$, we write $u v \in E$ and say that $u$ and $v$ are neighbours. The neighbourhood of a vertex $v \in V$ is the set of its neighbours; it is denoted by $N(v)$. The degree of $v$ is the size of its neighbourhood.

A subgraph of $G$ is spanning if it contains all vertices of $G$, and it is induced if two vertices of the subgraph are adjacent if and only if they are adjacent in $G$. If a graph $H$ is isomorphic to an induced subgraph of a graph $G$, we say that $G$ contains $H$. Otherwise we say that $G$ is $H$-free. Given a set of graphs $M$, we denote by $\operatorname{Free}(M)$ the set of all graphs containing no induced subgraphs from $M$.

A class of graphs (or graph property) is a set of graphs closed under isomorphism. A class is hereditary if it is closed under taking induced subgraphs. It is well-known (and not difficult to see) that a class $X$ is hereditary if and only if $X=\operatorname{Free}(M)$ for some set $M$. If $M$ is a finite set, we say that $X$ is finitely defined.

A class of graphs is monotone if it is closed under taking subgraphs (not necessarily induced). Clearly, every monotone class is hereditary.

In a graph, a clique is a subset of pairwise adjacent vertices, and an independent set is a subset of vertices no two of which are adjacent. A graph is bipartite if its vertices can be partitioned into two independent sets. It is well-known that a graph is bipartite if and only if it is free of odd cycles, i.e. if and only if it belongs to Free $\left(C_{3}, C_{5}, C_{7}, \ldots\right)$. We say that a graph $G$ is co-bipartite if $\bar{G}$ is bipartite. Clearly, a graph is co-bipartite if and only if it belongs to $\operatorname{Free}\left(\bar{C}_{3}, \bar{C}_{5}, \bar{C}_{7}, \ldots\right)$.

We complete this part of the section with the following technical lemma, proved in [1], where a private neighbour of a vertex $x \in D$ is a vertex $y \notin D$ such that $x$ is the only neighbour of $y$ in $D$.

Lemma 1. Let $G$ be a connected graph and $D$ a minimal dominating set in $G$. If there are vertices in $D$ that have no private neighbour outside of $D$, then $D$ 
can be transformed in polynomial time into a minimal dominating set $D^{\prime}$ with $\left|D^{\prime}\right| \leq|D|$ in which every vertex has a private neighbour outside of $D^{\prime}$.

\subsection{Boundary Classes of Graphs}

As we mentioned earlier, the notion of boundary classes of graphs was introduced in [2] to study the MAXIMUM INDEPENDENT SET problem in hereditary classes. Later this notion was applied to some other problems of both algorithmic $[3,4,13,17]$ and combinatorial $[14,15,19]$ nature. Assuming $P \neq N P$, the notion of boundary classes can be defined, with respect to algorithmic graph problems, as follows.

Let $\Pi$ be an algorithmic graph problem, which is generally NP-hard. We will say that a hereditary class $X$ of graphs is $\Pi$-tough if the problem is NP-hard for graphs in $X$ and $\Pi$-easy, otherwise. We define the notion of a boundary class for $\Pi$ in two steps. First, let us define the notion of a limit class.

Definition 1. A hereditary class $X$ is a LIMIT CLASS for $\Pi$ if $X$ is the intersection of a sequence $X_{1} \supseteq X_{2} \supseteq X_{3} \supseteq \ldots$ of $\Pi$-tough classes, in which case we also say that the sequence CONVERGES to $X$.

Example. To illustrate the notion of a limit class, let us quote a result from [20] stating that the MAXIMUM INDEPENDENT SET problem is NP-hard for graphs with large girth, i.e. for $\left(C_{3}, C_{4}, \ldots, C_{k}\right)$-free graphs for each fixed value of $k$. With $k$ tending to infinity, this sequence converges to the class of graphs without cycles, i.e. to forests. Therefore, the class of forests is a limit class for the MAXIMUM INDEPENDENT SET problem. However, this is not a minimal limit class for the problem, which can be explained as follows.

The proof of the NP-hardness of the problem for graphs with large girth is based on a simple fact that a double subdivision of an edge in a graph $G$ increases the size of a maximum independent set in $G$ by exactly 1 . This operation applied sufficiently many (but still polynomially many) times allows to destroy all small cycles in $G$, i.e. reduces the problem from an arbitrary graph $G$ to a graph $G^{\prime}$ of girth at least $k$. Obviously, if $G$ is a graph of vertex degree at most 3 , then so is $G^{\prime}$, and since the problem is NP-hard for graphs of degree at most 3, we conclude that it is also NP-hard for for $\left(C_{3}, C_{4}, \ldots, C_{k}\right)$-free graphs of degree at most 3 . This shows that the class of forests of vertex degree at most 3 is a limit class for the the MAXIMUM INDEPENDENT SET problem. However, it is still not a minimal limit class, because by the same operation (double subdivisions of edges) one can destroy small induced copies of the graph $H_{n}$ shown on the left of Fig. 1. Therefore, the MAXIMUM InDEPENDENT SET problem is NP-hard in the following class for each fixed value of $k$ :

$Z_{k}$ is the class of $\left(C_{3}, \ldots, C_{k}, H_{1}, \ldots, H_{k}\right)$-free graphs of degree at most 3 .

It is not difficult to see that the sequence $Z_{3} \supset Z_{4} \supset \ldots$ converges to the class of forests every connected component of which has the form $S_{i, j, \ell}$ represented on the right of Fig. 1. Throughout the paper we denote this class by $\mathcal{S}$, i.e. $\mathcal{S}$ is the intersection of the sequence $Z_{3} \supset Z_{4} \supset \ldots$ 

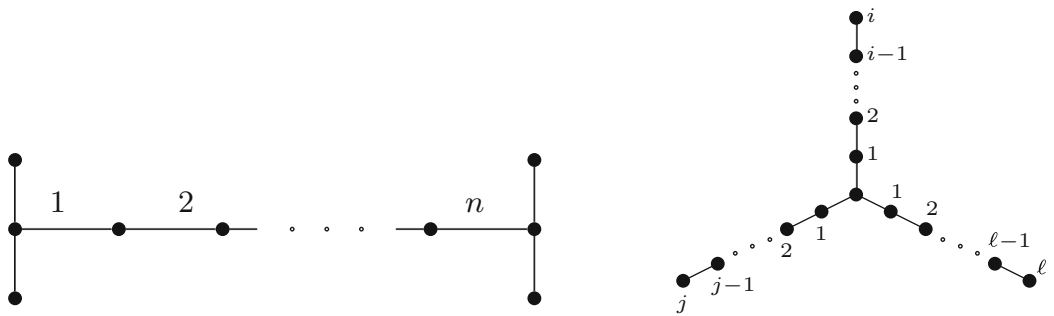

Fig. 1. Graphs $H_{n}$ (left) and $S_{i, j, \ell}$ (right).

The above discussion shows that $\mathcal{S}$ is a limit class for the MAXIMUM INDEPENDENT SET problem. Moreover, in [2] it was proved that $S$ is a minimal limit class for this problem.

Definition 2. A minimal (with respect to set inclusion) limit class for a problem $\Pi$ is called a boundary class for $\Pi$.

The importance of the notion of boundary classes for NP-hard algorithmic graph problems is due to the following theorem proved originally for the MAXIMUM INDEPENDENT SET problem in [2] (can also be found in [3] in a more general context).

Theorem 1. If $\mathrm{P} \neq \mathrm{NP}$, then a finitely defined class $X$ is $\Pi$-tough if and only if $X$ contains a boundary class for $\Pi$.

In what follows, we identify the first boundary class for the UPPER DOMINATING SET problem. To this end, we need a number of auxiliary results. The first of them is the following lemma dealing with limit classes, which was derived in $[2,3]$ as a step towards the proof of Theorem 1.

Lemma 2. If $X$ is a finitely defined class containing a limit class for an NPhard problem $\Pi$, then $X$ is $\Pi$-tough.

The next two results were proved in [12] and [3], respectively.

Lemma 3. The Minimum DOMINATING SET problem is NP-hard in the class $Z_{k}$ for each fixed value of $k$.

Theorem 2. The class $\mathcal{S}$ is a boundary class for MINIMUM DOMINATING SET problem.

\section{A Boundary Class for Upper Domination}

To describe a boundary class for the UPPER DOMINATING SET problem, let us introduce the following graph transformations. Given a graph $G=(V, E)$, we denote by 
$S(G)$ the incidence graph of $G$, i.e. the graph with vertex set $V \cup E$, where $V$ and $E$ are independent sets and a vertex $v \in V$ is adjacent to a vertex $e \in E$ in $S(G)$ if and only if $v$ is incident to $e$ in $G$. Alternatively, $S(G)$ is obtained from $G$ by subdividing each edge $e$ by a new vertex $v_{e}$. According to this interpretation, we call $E$ the set of new vertices and $V$ the set of old vertices. Any graph of the form $S(G)$ for some $G$ will be called a subdivision graph.

$Q(G)$ the graph obtained from $S(G)$ by creating a clique on the set of old vertices and a clique on the set of new vertices. We call any graph of the form $Q(G)$ for some $G$ a $Q$-graph.

The importance of $Q$-graphs for the UPPER DOMINATING SET problem is due to the following lemma, where we denote by $\Gamma(G)$ the size of an upper dominating set in $G$ and by $\gamma(G)$ the size of a dominating set of minimum cardinality in $G$.

Lemma 4. Let $G$ be a graph with $n$ vertices such that $\Gamma(Q(G)) \geq 3$. Then $\Gamma(Q(G))=n-\gamma(G)$.

Proof. Let $D$ be a minimum dominating set in $G$, i.e. a dominating set of size $\gamma(G)$. Without loss of generality, we will assume that $D$ satisfies Lemma 1, i.e. every vertex of $D$ has a private neighbour outside of $D$. For every vertex $u$ outside of $D$, consider exactly one edge, chosen arbitrarily, connecting $u$ to a vertex in $D$ and denote this edge by $e_{u}$. We claim that the set $D^{\prime}=\left\{v_{e_{u}}: u \notin D\right\}$ is a minimal dominating set in $Q(G)$. By construction, $D^{\prime}$ dominates $E \cup(V-D)$ in $Q(G)$. To show that it also dominates $D$, assume by contradiction that a vertex $w \in D$ is not dominated by $D^{\prime}$ in $Q(G)$. By Lemma 1 we know that $w$ has a private neighbour $u$ outside of $D$. But then the edge $e=u w$ is the only edge connecting $u$ to a vertex in $D$. Therefore, $v_{e}$ necessarily belongs to $D^{\prime}$ and hence it dominates $w$, contradicting our assumption. In order to show that $D^{\prime}$ is a minimal dominating set, we observe that if we remove from $D^{\prime}$ a vertex $v_{e_{u}}$ with $e_{u}=u w, u \notin D, w \in D$, then $u$ becomes undominated in $Q(G)$. Finally, since $\left|D^{\prime}\right|=n-|D|$, we conclude that $\Gamma(Q(G)) \geq n-|D|=n-\gamma(G)$.

Conversely, let $D^{\prime}$ be an upper dominating set in $Q(G)$, i.e. a minimal dominating set of size $\Gamma(Q(G)) \geq 3$. Then $D^{\prime}$ cannot intersect both $V$ and $E$, since otherwise it contains exactly one vertex in each of these sets (else it is not minimal, because each of these sets is a clique), in which case $\left|D^{\prime}\right|=2$.

Assume first that $D^{\prime} \subseteq V$. Then $V-D^{\prime}$ is an independent set in $G$. Indeed, if $G$ contains an edge $e$ connecting two vertices in $V-D^{\prime}$, then vertex $v_{e}$ is not dominated by $D^{\prime}$ in $Q(G)$, a contradiction. Moreover, $V-D^{\prime}$ is a maximal (with respect to set-inclusion) independent set in $G$, because $D^{\prime}$ is a minimal dominating set in $Q(G)$. Therefore, $V-D^{\prime}$ is a dominating set in $G$ of size $n-\Gamma(Q(G))$ and hence $\gamma(G) \leq n-\Gamma(Q(G))$.

Now assume $D^{\prime} \subseteq E$. Let us denote by $G^{\prime}$ the subgraph of $G$ formed by the edges (and all their endpoints) $e$ such that $v_{e} \in D^{\prime}$. Then:

- $G^{\prime}$ is a spanning forest of $G$, because $D^{\prime}$ covers $V$ (else $D^{\prime}$ is not dominating in $Q(G)$ ) and $G^{\prime}$ is acyclic (else $D^{\prime}$ is not a minimal dominating set in $Q(G)$ ). 
- $G^{\prime}$ is $P_{4}$-free, i.e. each connected component of $G^{\prime}$ is a star, since otherwise $D^{\prime}$ is not a minimal dominating set in $Q(G)$, because any vertex of $D^{\prime}$ corresponding to the middle edge of a $P_{4}$ in $G^{\prime}$ can be removed from $D^{\prime}$.

Let $D$ be the set of the centers of the stars of $G^{\prime}$. Then $D$ is dominating in $G$ (since $D^{\prime}$ covers $V$ ) and $|D|=n-\left|D^{\prime}\right|$, i.e. $\gamma(G) \leq n-\Gamma(Q(G)$ ), as required.

Lemma 4 together with Theorem 2 suggest the following natural idea about a boundary class for the UPPER DOMINATING SET problem: it is the class of graphs $Q(G)$ obtained from graphs $G$ in $\mathcal{S}$. In order to transform this idea into a formal proof, we need more notations and more auxiliary results.

For an arbitrary class $X$ of graphs, we denote $S(X):=\{S(G): G \in X\}$ and $Q(X):=\{Q(G): G \in X\}$. In particular, $Q(\mathcal{G})$ is the set of all $Q$-graphs, where $\mathcal{G}$ is the class of all simple graphs. We observe that an induced subgraph of a $Q$-graph is not necessarily a $Q$-graph. Indeed, in a $Q$-graph every new vertex is adjacent to exactly two old vertices. However, by deleting some old vertices in a $Q$-graph we may obtain a graph in which a new vertex is adjacent to at most one old vertex. Therefore, $Q(X)$ is not necessarily hereditary even if $X$ is a hereditary class. We denote by $Q^{*}(X)$ the hereditary closure of $Q(X)$, i.e. the class obtained from $Q(X)$ by adding to it all induced subgraphs of the graphs in $Q(X)$. Similarly, we denote by $S^{*}(X)$ the hereditary closure of $S(X)$.

With the above notation, our goal is proving that $Q^{*}(\mathcal{S})$ is a boundary class for the UPPER DOMINATING SET problem. To achieve this goal we need the following lemmas.

Lemma 5. Let $X$ be a monotone class of graphs such that $\mathcal{S} \nsubseteq X$, then the clique-width of the graphs in $Q^{*}(X)$ is bounded by a constant.

Proof. In [16], it was proved that if $\mathcal{S} \nsubseteq X$, then the clique-width is bounded for graphs in $X$. It is known (see e.g. [8]) that for monotone classes, the clique-width is bounded if and only if the tree-width is bounded. By subdividing the edges of all graphs in $X$ exactly once, we transform $X$ into the class $S(X)$, where the tree-width is still bounded, since the subdivision of an edge of a graph does not change its tree-width. Since bounded tree-width implies bounded clique-width (see e.g. [8]), we conclude that $S(X)$ is a class of graphs of bounded cliquewidth. Now, for each graph $G$ in $S(X)$ we create two cliques by complementing the edges within the sets of new and old vertices. This transforms $S(X)$ into $Q(X)$. It is known (see e.g. [11]) that local complementations applied finitely many times do not change the clique-width "too much", i.e. they transform a class of graphs of bounded clique-width into another class of graphs of bounded clique-width. Therefore, the clique-width of graphs in $Q(X)$ is bounded. Finally, the clique-width of a graph is never smaller than the clique-width of any of its induced subgraphs (see e.g. [8]). Therefore, the clique-width of graphs in $Q^{*}(X)$ is also bounded.

Lemma 6. Let $X \subseteq Q^{*}(\mathcal{G})$ be a hereditary class. The clique-width of graphs in $X$ is bounded by a constant if and only if it is bounded for Q-graphs in $X$. 
Proof. The lemma is definitely true if $X=Q^{*}(Y)$ for some class $Y$. In this case, by definition, every non- $Q$-graph in $X$ is an induced subgraph of a $Q$-graph from $X$. However, in general, $X$ may contain a non- $Q$-graph $H$ such that no $Q$-graph containing $H$ as an induced subgraph belongs to $X$. In this case, we prove the result as follows.

First, we transform each graph $H$ in $X$ into a bipartite graph $H^{\prime}$ by replacing the two cliques of $H$ (i.e. the sets of old and new vertices) with independent sets. In this way, $X$ transforms into a class $X^{\prime}$ which is a subclass of $S^{*}(\mathcal{G})$. As we mentioned in the proof of Lemma 5, this transformation does not change the clique-width "too much", i.e. the clique-width of graphs in $X$ is bounded if and only if it is bounded for graphs in $X^{\prime}$.

By definition, $H \in X$ is a $Q$-graph if and only if $H^{\prime}$ is a subdivision graph, i.e. $H^{\prime}=S(G)$ for some graph $G$. Therefore, we need to show that the clique-width of graphs in $X^{\prime}$ is bounded if and only if it is bounded for subdivision graphs in $X^{\prime}$. In one direction, the statement is trivial. To prove it in the other direction, assume the clique-width of subdivision graphs in $X^{\prime}$ is bounded. If $H^{\prime}$ is not a subdivision, it contains new vertices of degree 0 or 1 . If $H^{\prime}$ contains a vertex of degree 0 , then it is disconnected, and if $H^{\prime}$ contains a vertex $x$ of degree 1 , then it has a cut-point (the neighbour of $x$ ). It is well-known that the cliquewidth of graphs in a hereditary class is bounded if and only if it is bounded for connected graphs in the class. Moreover, it was shown in [18] that the cliquewidth of graphs in a hereditary class is bounded if and only if it is bounded for 2-connected graphs (i.e. connected graphs without cut-points) in the class. Since connected graphs without cut-points in $X^{\prime}$ are subdivision graphs, we conclude that the clique-width is bounded for all graphs in $X^{\prime}$.

Finally, to prove the main result of this paper, we need to show that $Q^{*}(\mathcal{G})$ is a finitely defined class. To show this, we first characterize graphs in $Q^{*}(\mathcal{G})$ as follows: a graph $G$ belongs to $Q^{*}(\mathcal{G})$ if and only if the vertices of $G$ can be partitioned into two (possibly empty) cliques $U$ and $W$ such that

(a) every vertex in $W$ has at most two neighbours in $U$,

(b) if $x$ and $y$ are two vertices of $W$ each of which has exactly two neighbours in $U$, then $N(x) \cap U \neq N(y) \cap U$.

In the proof of the following lemma, we call any partition satisfying (a) and (b) nice. Therefore, $Q^{*}(\mathcal{G})$ is precisely the class of graphs admitting a nice partition. Now we characterize $Q^{*}(\mathcal{G})$ in terms of minimal forbidden induced subgraphs.

Lemma 7. $Q^{*}(\mathcal{G})=$ Free $(N)$, where $N$ is the set of eleven graphs consisting of $\bar{C}_{3}, \bar{C}_{5}, \bar{C}_{7}$ and the eight graphs shown in Fig. 2.

Proof. To show the inclusion $Q^{*}(\mathcal{G}) \subseteq \operatorname{Free}(N)$, we first observe that $\bar{C}_{3}, \bar{C}_{5}$ and $\bar{C}_{7}$ are forbidden in $Q^{*}(\mathcal{G})$, since every graph in this class is co-bipartite, while $\bar{C}_{3}, \bar{C}_{5}, \bar{C}_{7}$ are not co-bipartite. Each of the remaining eight graphs of the set $N$ is co-bipartite, but none of them admits a nice partition, which is a routine matter to check. 


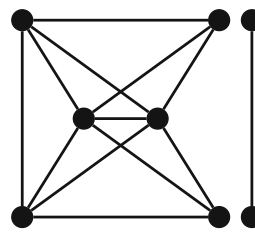

$G_{1}$

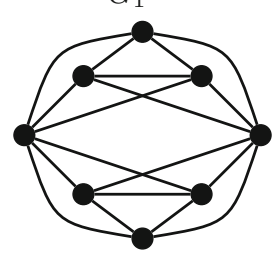

$G_{5}$

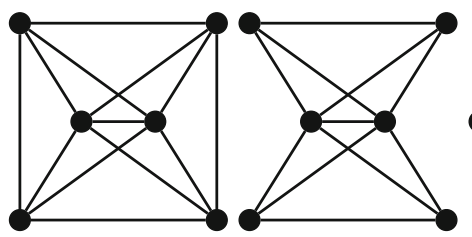

$G_{3}$

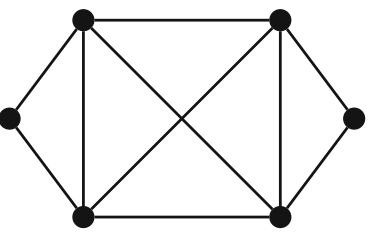

$G_{4}$

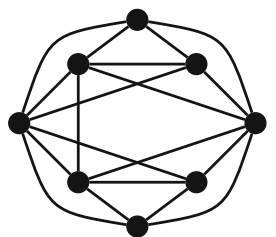

$G_{6}$

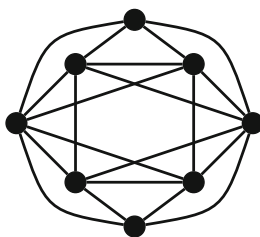

$G_{7}$

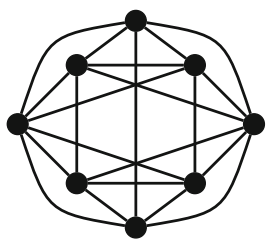

$G_{8}$

Fig. 2. Forbidden graphs for $Q^{*}(\mathcal{G})$

To prove the inverse inclusion $\operatorname{Free}(N) \subseteq Q^{*}(\mathcal{G})$, let us consider a graph $G$ in Free $(N)$. By definition, $G$ contains no $\bar{C}_{3}, \bar{C}_{5}, \bar{C}_{7}$. Also, since $G_{1}$ is an induced subgraph of $\bar{C}_{i}$ with $i \geq 9$, we conclude that $G$ contains no complements of odd cycles of length 9 or more. Therefore, $G$ is co-bipartite. Let $V_{1} \cup V_{2}$ be an arbitrary bipartition of $V(G)$ into two cliques. In order to show that $G$ belongs to $Q^{*}(\mathcal{G})$, we split our analysis into several cases.

Case 1: $G$ contains a $K_{4}$ induced by vertices $x_{1}, y_{1} \in V_{1}$ and $x_{2}, y_{2} \in V_{2}$. To analyze this case, we partition the vertices of $V_{1}$ into four subsets with respect to $x_{2}, y_{2}$ as follows:

$A_{1}$ is the set of vertices of $V_{1}$ adjacent to $x_{2}$ and non-adjacent to $y_{2}$, $B_{1}$ is the set of vertices of $V_{1}$ adjacent to $x_{2}$ and to $y_{2}$,

$C_{1}$ is the set of vertices of $V_{1}$ adjacent to $y_{2}$ and non-adjacent to $x_{2}$,

$D_{1}$ is the set of vertices of $V_{1}$ adjacent neither to $x_{2}$ nor to $y_{2}$.

We partition the vertices of $V_{2}$ with respect to $x_{1}, y_{1}$ into four subsets $A_{2}, B_{2}$, $C_{2}, D_{2}$ analogously. We now observe the following.

(1) For $i \in\{1,2\}$, either $A_{i}=\emptyset$ or $C_{i}=\emptyset$, since otherwise a vertex in $A_{i}$ and a vertex in $C_{i}$ together with $x_{1}, y_{1}, x_{2}, y_{2}$ induce $G_{2}$.

According to this observation, in what follows, we may assume, without loss of generality, that

$-C_{1}=\emptyset$ and $C_{2}=\emptyset$.

We next observe that

(2) Either $A_{1}=\emptyset$ or $A_{2}=\emptyset$, since otherwise a vertex $a_{1} \in A_{1}$ and a vertex $a_{2} \in A_{2}$ together with $x_{1}, y_{1}, x_{2}, y_{2}$ induce either $G_{1}$ (if $a_{1}$ is not adjacent to $a_{2}$ ) or $G_{2}$ (if $a_{1}$ is adjacent to $a_{2}$ ). 
Observation (2) allows us to assume, without loss of generality, that

$-A_{2}=\emptyset$.

We further make the following conclusions:

(3) For $i \in\{1,2\},\left|D_{i}\right| \leq 1$, since otherwise any two vertices of $D_{i}$ together with $x_{1}, x_{2}, y_{1}, y_{2}$ induce $G_{3}$.

(4) If $D_{1}=\left\{d_{1}\right\}$ and $D_{2}=\left\{d_{2}\right\}$, then $d_{1}$ is adjacent to $d_{2}$, since otherwise $d_{1}, d_{2}, x_{1}, x_{2}, y_{1}, y_{2}$ induce $G_{4}$.

(5) If $A_{1} \cup D_{1} \cup D_{2} \neq \emptyset$, then every vertex of $B_{1}$ is adjacent to every vertex of $B_{2}$. Indeed, assume, without loss of generality, that $z \in A_{1} \cup D_{1}$ and a vertex $b_{1} \in B_{1}$ is not adjacent to a vertex $b_{2} \in B_{2}$. Then the vertices $z, b_{1}, b_{2}, x_{1}, x_{2}, y_{1}$ induce either $G_{1}$ (if $z$ is not adjacent to $b_{2}$ ) or $G_{2}$ (if $z$ is adjacent to $b_{2}$ ).

(6) Either $A_{1}=\emptyset$ or $D_{1}=\emptyset$, since otherwise a vertex in $A_{1}$ and a vertex in $D_{1}$ together with $x_{1}, y_{1}, x_{2}, y_{2}$ induce $G_{1}$.

According to (6), we split our analysis into three subcases as follows.

Case 1.1: $D_{1}=\left\{d_{1}\right\}$. Then $A_{1}=\emptyset$ (by (6)) and every vertex of $B_{1}$ is adjacent to every vertex of $B_{2}$ (by (5)). If $D_{2}=\emptyset$, then $U=D_{1}$ and $W=B_{1} \cup B_{2}$ is a nice partition of $G$ (remember that $x_{1}, y_{1} \in B_{1}$ and $x_{2}, y_{2} \in B_{2}$ ).

Now assume $D_{2}=\left\{d_{2}\right\}$ and denote by $B_{1}^{0}$ the vertices of $B_{1}$ nonadjacent to $d_{2}$ and by $B_{1}^{1}$ the vertices of $B_{1}$ adjacent to $d_{2}$. Similarly, we denote by $B_{2}^{0}$ the vertices of $B_{2}$ nonadjacent to $d_{1}$ and by $B_{2}^{1}$ the vertices of $B_{2}$ adjacent to $d_{1}$. Then $\left|B_{1}^{1} \cup B_{2}^{1}\right| \leq 1$, since otherwise any two vertices of $B_{1}^{1} \cup B_{2}^{1}$ together with $x_{1}, x_{2}, d_{1}, d_{2}$ induce $G_{2}$. But then $U=D_{1} \cup D_{2}$ and $W=B_{1} \cup B_{2}$ is a nice partition of $G$.

Case 1.2: $A_{1} \neq \emptyset$. Then $D_{1}=\emptyset$ (by (6)) and every vertex of $B_{1}$ is adjacent to every vertex of $B_{2}$ (by (5)). In this case, we claim that

(7) every vertex of $B_{2}$ is either adjacent to every vertex of $A_{1}$ or to none of them. Indeed, assume a vertex $b_{2} \in B_{2}$ has a neighbour $a^{\prime} \in A_{1}$ and a non-neighbour $a^{\prime \prime} \in A_{1}$. Then $b_{2}, a^{\prime}, a^{\prime \prime}, x_{1}, y_{1}, y_{2}$ induce $G_{1}$.

We denote by $B_{2}^{0}$ the subset of vertices of $B_{2}$ that have no neighbours in $A_{1}$ and by $B_{2}^{1}$ the subset of vertices of $B_{2}$ adjacent to every vertex of $A_{1}$. Then

- either $\left|A_{1}\right|=1$ or $\left|B_{2}^{0}\right|=1$, since otherwise any two vertices of $A_{1}$ together with any two vertices of $B_{2}^{0}$ and any two vertices of $B_{1}$ induce $G_{3}$.

- if $D_{2}=\left\{d_{2}\right\}$, then $\left|B_{2}^{1}\right|=1$, since otherwise any two vertices of $B_{2}^{1}$ together with $d_{2}, x_{1}, y_{2}$ and any vertex $a$ in $A_{1}$ induce either $G_{1}$ (if $a$ is not adjacent to $\left.d_{2}\right)$ ) or $G_{2}$ (if $a$ is adjacent to $\left.d_{2}\right)$ ).

- if $D_{2}=\left\{d_{2}\right\}$, then $d_{2}$ has no neighbours in $B_{1}$. Indeed, if $d_{2}$ has a neighbour $b_{1} \in B_{1}$, then vertices $b_{1}, d_{2}, x_{1}, x_{2}, y_{2}$ together with any vertex $a_{1} \in A_{1}$ induce either $G_{1}$ (if $d_{2}$ is not adjacent to $a_{1}$ ) or $G_{2}$ (if $d_{2}$ is adjacent to $a_{1}$ ). 
Therefore, either $U=A_{1} \cup D_{2}, W=B_{1} \cup B_{2}$ (if $\left|A_{1}\right|=1$ ) or $U=B_{2}^{0} \cup D_{2}$, $W=A_{1} \cup B_{1} \cup B_{2}^{1}$ (if $\left|B_{2}^{0}\right|=1$ ) is a nice partition of $G$.

Case 1.3: $A_{1}=\emptyset$ and $D_{1}=\emptyset$. In this case, if $D_{2} \neq \emptyset$, then $U=D_{2}$, $W=B_{1} \cup B_{2}$ is a nice partition of $G$, since $B_{1} \cup B_{2}$ is a clique (by (5)). Assume now that $D_{2}=\emptyset$. If $B_{1} \cup B_{2}$ is a clique, then $G$ has a trivial nice partition. Suppose next that $B_{1} \cup B_{2}$ is not a clique. If all non-edges of $G$ are incident to a same vertex, say $b$ (i.e. $b$ is incident to all the edges of $\bar{G}$ ), then $U=\{b\}$, $W=\left(B_{1} \cup B_{2}\right)-\{b\}$ is a nice partition of $G$. Otherwise, $G$ contains a pair of nonedges $b_{1}^{\prime} b_{2}^{\prime} \notin E(G)$ and $b_{1}^{\prime \prime} b_{2}^{\prime \prime} \notin E(G)$ with all four vertices $b_{1}^{\prime}, b_{1}^{\prime \prime} \in B_{1}, b_{2}^{\prime}, b_{2}^{\prime \prime} \in$ $B_{2}$ being distinct (i.e. $b_{1}^{\prime} b_{2}^{\prime}$ and $b_{1}^{\prime \prime} b_{2}^{\prime \prime}$ form a matching in $\bar{G}$ ). We observe that $\left\{b_{1}^{\prime}, b_{1}^{\prime \prime}, b_{2}^{\prime}, b_{2}^{\prime \prime}\right\} \cap\left\{x_{1}, y_{1}, x_{2}, y_{2}\right\}=\emptyset$, because by definition vertices $x_{1}, y_{1}, x_{2}, y_{2}$ dominate the set $B_{1} \cup B_{2}$. But then $b_{1}^{\prime}, b_{1}^{\prime \prime}, b_{2}^{\prime}, b_{2}^{\prime \prime}, x_{1}, y_{1}$ induce either $G_{2}$ (if both $b_{1}^{\prime} b_{2}^{\prime \prime}$ and $b_{2}^{\prime} b_{1}^{\prime \prime}$ are edges in $G$ ) or $G_{1}$ (if exactly one of $b_{1}^{\prime} b_{2}^{\prime \prime}$ and $b_{2}^{\prime} b_{1}^{\prime \prime}$ is an edge in $G$ ) or $G_{3}$ (if neither $b_{1}^{\prime} b_{2}^{\prime \prime}$ nor $b_{2}^{\prime} b_{1}^{\prime \prime}$ is an edge in $G$ ). This completes the proof of Case 1.

Case 2: $G$ contains no $K_{4}$ with two vertices in $V_{1}$ and two vertices in $V_{2}$. We claim that in this case $V_{1} \cup V_{2}$ is a nice partition of $G$. First, the assumption of case 2 implies that that no two vertices in the same part of the bipartition $V_{1} \cup V_{2}$ have two common neighbours in the opposite part, verifying condition (b) of the definition of nice partition. To verify condition (a), it remains to prove that one of the parts $V_{1}$ and $V_{2}$ has no vertices with more than two neighbours in the opposite part. Assume the contrary and let $a_{1} \in V_{1}$ have three neighbours in $V_{2}$ and let $a_{2} \in V_{2}$ have three neighbours in $V_{1}$.

First, suppose $a_{1}$ is adjacent to $a_{2}$. Denote by $b_{2}, c_{2}$ two other neighbours of $a_{1}$ in $V_{2}$ and by $b_{1}, c_{1}$ two other neighbours of $a_{2}$ in $V_{1}$. Then there are no edges between $b_{1}, c_{1}$ and $b_{2}, c_{2}$, since otherwise we are in conditions of Case 1. But now $a_{1}, b_{1}, c_{1}, a_{2}, b_{2}, c_{2}$ induce a $G_{3}$.

Suppose now that $a_{1}$ is not adjacent to $a_{2}$. We denote by $b_{2}, c_{2}, d_{2}$ three neighbours of $a_{1}$ in $V_{2}$ and by $b_{1}, c_{1}, d_{1}$ three neighbours of $a_{2}$ in $V_{1}$. No two edges between $b_{1}, c_{1}, d_{1}$ and $b_{2}, c_{2}, d_{2}$ (if any) share a vertex, since otherwise we are in conditions of Case 1 . But then $a_{1}, b_{1}, c_{1}, d_{1}, a_{2}, b_{2}, c_{2}, d_{2}$ induce either $G_{5}$ or $G_{6}$ or $G_{7}$ or $G_{8}$. This contradiction completes the proof of the lemma.

Now we are ready to prove the main result of the paper.

Theorem 3. If $\mathrm{P} \neq \mathrm{NP}$, then $Q^{*}(\mathcal{S})$ is a boundary class for the UPPER DOMINATING SET problem.

Proof. From Lemmas 3 and 4 we know that UPPER DOMINATION is NP-hard in the class $Q^{*}\left(Z_{k}\right)$ for all values of $k \geq 3$. Also, it is not hard to verify that the sequence of classes $Q^{*}\left(Z_{1}\right), Q^{*}\left(Z_{2}\right) \ldots$ converges to $Q^{*}(\mathcal{S})$. Therefore, $Q^{*}(\mathcal{S})$ is a limit class for the UPPER DOMINATING SET problem. To prove its minimality, assume there is a limit class $X$ which is properly contained in $Q^{*}(\mathcal{S})$. We consider a graph $F \in Q^{*}(\mathcal{S})-X$, a graph $G \in Q(\mathcal{S})$ containing $F$ as an induced subgraph (possibly $G=F$ if $F \in Q(\mathcal{S})$ ) and a graph $H \in \mathcal{S}$ such that $G=Q(H)$. 
From the choice of $G$ and Lemma 7, we know that $X \subseteq \operatorname{Free}(N \cup\{G\})$, where $N$ is the set of minimal forbidden induced subgraphs for the class $Q^{*}(\mathcal{G})$. Since the set $N$ is finite (by Lemma 7 ), we conclude with the help of Lemma 2 that the UPPER DOMINATING SET problem is NP-hard in the class Free $(N \cup\{G\})$. To obtain a contradiction, we will show that graphs in Free $(N \cup\{G\})$ have bounded clique-width.

Denote by $M$ the set of all graphs containing $H$ as a spanning subgraph. Clearly Free $(M)$ is a monotone class. More precisely, it is the class of graphs containing no $H$ as a subgraph (not necessarily induced). Since Free $(M)$ is monotone and $\mathcal{S} \not \subset$ Free $(\mathcal{M})$ (as $H \in \mathcal{S}$ ), we know from Lemma 5 that the clique-width is bounded in $Q^{*}($ Free $(M))$.

To prove that graphs in Free $(N \cup\{G\})$ have bounded clique-width, we will show that $Q$-graphs in this class belong to $Q^{*}(\operatorname{Free}(M))$. Let $Q\left(H^{\prime}\right)$ be a $Q$ graph in Free $(N \cup\{G\})$. Since the vertices of $Q\left(H^{\prime}\right)$ represent the vertices and the edges of $H^{\prime}$ and $Q\left(H^{\prime}\right)$ does not contain $G$ as an induced subgraph, we conclude that $H^{\prime}$ does not contain $H$ as a subgraph. Therefore, $H^{\prime} \in \operatorname{Free}(M)$, and hence $Q\left(H^{\prime}\right) \in Q(\operatorname{Free}(M))$. By Lemma 6, this implies that all graphs in Free $(N \cup\{G\})$ have bounded clique-width. This contradicts the fact that the UPPER DOMINATING SET problem is NP-hard in this class and completes the proof of the theorem.

\section{Conclusion}

In this paper, we identified the first boundary class for the UPPER DOMINATING SET problem. Since the problem is NP-hard in the class of triangle-free graphs [1], we known (by Theorem 1) that there must exist at least one more boundary class for the problem. Revealing this class is a challenging open question.

\section{References}

1. AbouEisha, H., Hussain, S., Lozin, V., Monnot, J., Ries, B.: A dichotomy for upper domination in monogenic classes. In: Zhang, Z., Wu, L., Xu, W., Du, D.-Z. (eds.) COCOA 2014. LNCS, vol. 8881, pp. 258-267. Springer, Heidelberg (2014)

2. Alekseev, V.E.: On easy and hard hereditary classes of graphs with respect to the independent set problem. Discrete Appl. Math. 132, 17-26 (2003)

3. Alekseev, V.E., Korobitsyn, D.V., Lozin, V.V.: Boundary classes of graphs for the dominating set problem. Discrete Math. 285, 1-6 (2004)

4. Alekseev, V.E., Boliac, R., Korobitsyn, D.V., Lozin, V.V.: NP-hard graph problems and boundary classes of graphs. Theor. Comput. Sci. 389, 219-236 (2007)

5. Cheston, G.A., Fricke, G., Hedetniemi, S.T., Jacobs, D.P.: On the computational complexity of upper fractional domination. Discrete Appl. Math. 27(3), 195-207 (1990)

6. Cockayne, E.J., Favaron, O., Payan, C., Thomason, A.G.: Contributions to the theory of domination, independence and irredundance in graphs. Discrete Math. 33(3), 249-258 (1981) 
7. Courcelle, B., Makowsky, J.A., Rotics, U.: Linear time solvable optimization problems on graphs of bounded clique-width. Theor. Comput. Syst. 33(2), 125-150 (2000)

8. Courcelle, B., Olariu, S.: Upper bounds to the clique-width of a graph. Discrete Appl. Math. 101, 77-114 (2000)

9. Hare, E.O., Hedetniemi, S.T., Laskar, R.C., Peters, K., Wimer, T.: Linear-time computability of combinatorial problems on generalized-series-parallel graphs. In: Johnson, D.S., et al. (eds.) Discrete Algorithms and Complexity, pp. 437-457. Academic Press, New York (1987)

10. Jacobson, M.S., Peters, K.: Chordal graphs and upper irredundance, upper domination and independence. Discrete Math. 86(1-3), 59-69 (1990)

11. Kamiński, M., Lozin, V., Milanič, M.: Recent developments on graphs of bounded clique-width. Discrete Appl. Math. 157, 2747-2761 (2009)

12. Korobitsyn, D.V.: On the complexity of determining the domination number in monogenic classes of graphs. Diskretnaya Matematika 2(3), 90-96 (1990). (in Russian, translation in Discrete Math. Appl. 2(2), 191-199 (1992))

13. Korpelainen, N., Lozin, V.V., Malyshev, D.S., Tiskin, A.: Boundary properties of graphs for algorithmic graph problems. Theor. Comput. Sci. 412, 3545-3554 (2011)

14. Korpelainen, N., Lozin, V., Razgon, I.: Boundary properties of well-quasi-ordered sets of graphs. Order 30, 723-735 (2013)

15. Lozin, V.V.: Boundary classes of planar graphs. Comb. Probab. Comput. 17, 287$295(2008)$

16. Lozin, V., Milanič, M.: Critical properties of graphs of bounded clique-width. Discrete Math. 313, 1035-1044 (2013)

17. Lozin, V., Purcell, C.: Boundary properties of the satisfiability problems. Inf. Process. Lett. 113, 313-317 (2013)

18. Lozin, V., Rautenbach, D.: On the band-, tree- and clique-width of graphs with bounded vertex degree. SIAM J. Discrete Math. 18, 195-206 (2004)

19. Lozin, V., Zamaraev, V.: Boundary properties of factorial classes of graphs. J. Graph Theor. 78, 207-218 (2015)

20. Murphy, O.J.: Computing independent sets in graphs with large girth. Discrete Appl. Math. 35, 167-170 (1992) 